\title{
An immune stress test for resilience to aging: Pneumococcal vaccine response
}

\author{
Ryan Oveson ${ }^{\mathrm{a}}$, Zhou Jiang ${ }^{\mathrm{a}}$, Michali Izhaky ${ }^{\mathrm{a}}$, Kavita Sharma ${ }^{\mathrm{a}}$, Warren C. Ladiges ${ }^{\mathrm{a}, *}$ \\ ${ }^{a}$ Department of Comparative Medicine, School of Medicine, University of Washington, Seattle, WA, USA.
}

\begin{abstract}
The idea that the degree of response to physical stress in early life can be used to measure health in later life is a novel approach to better define resilience to aging. To investigate this, middle-age (15 months) mice were stressed by vaccination with a commercial pneumococcal vaccine (Prevnar 13), and 30 days later separated into a high antibody response group and a low antibody response group using Elisa to detect IgG serum antibody levels. After 4 months, mice were evaluated for physiological performance and learning ability. The high antibody response group was able to stay on a rotating rod longer than the low antibody response group and were more quickly able to find the escape hole in a spatial navigation learning task. This observation suggests Prevnar 13 antibody response in midlife could be a useful stress test to predict healthy aging.

Keywords: Resilience to aging, immune stress test, prevnar 13 vaccine, elisa antibody titers, aging mice
\end{abstract}

Resilience to aging is a well-recognized biological event that precedes age-related decline in physiological function and is defined as an organism's ability to respond to physical stressors with increasing age [1-2]. However, predictive factors of physical resilience for maintaining optimum health with increasing age have not been well characterized. One type of stress on the immune system is vaccination, which can be measured by the degree of antibody response. As a preclinical model to investigate this approach, 15-month old C57BL/6 mice were vaccinated with a 1:10 dilution of Prevnar 13 (Pfizer), a commonly used pneumococcal vaccine in older people. Blood was collected 30 days after vaccination and a Prevnar 13-specific mouse IgG antibody ELISA kit (Invitrogen, Thermo Fisher Scientific) was used to quantify serum IgG antibody levels. Five months following vaccination, mice were tested for physiological performance in a spatial navigation task for learning ability and a rotating rod for motor coordination. Mice could be grouped into high responders and low responders based on the strength of detecting $\operatorname{IgG}$ antibody levels to the Prevnar 13 vaccine (Figure 1).

Five months following Prevnar 13 vaccination, mice in

* Corresponding author: Warren Ladiges

Mailing address: Department of Comparative Medicine, School of Medicine, University of Washington, Seattle, WA, USA.

Email: wladiges@uw.edu

Received:15 September 2020 / Accepted: 21 September 2020

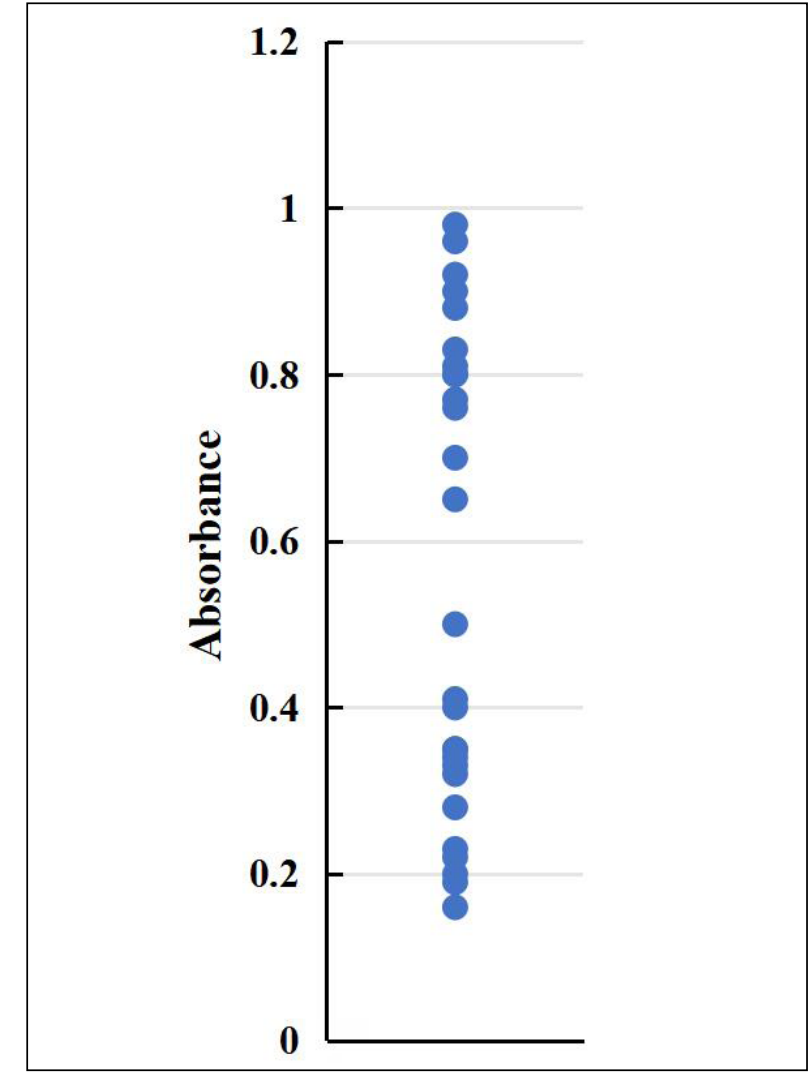

Figure 1. Mice injected subcutaneously with a 1:10 dilution of Prevnar 13 vaccine could be separated into high and low responders based on strength of Elisa antibody levels 30 days after vaccination, $N=25$. The cutoff between high and low responders was 0.562 absorbance. 


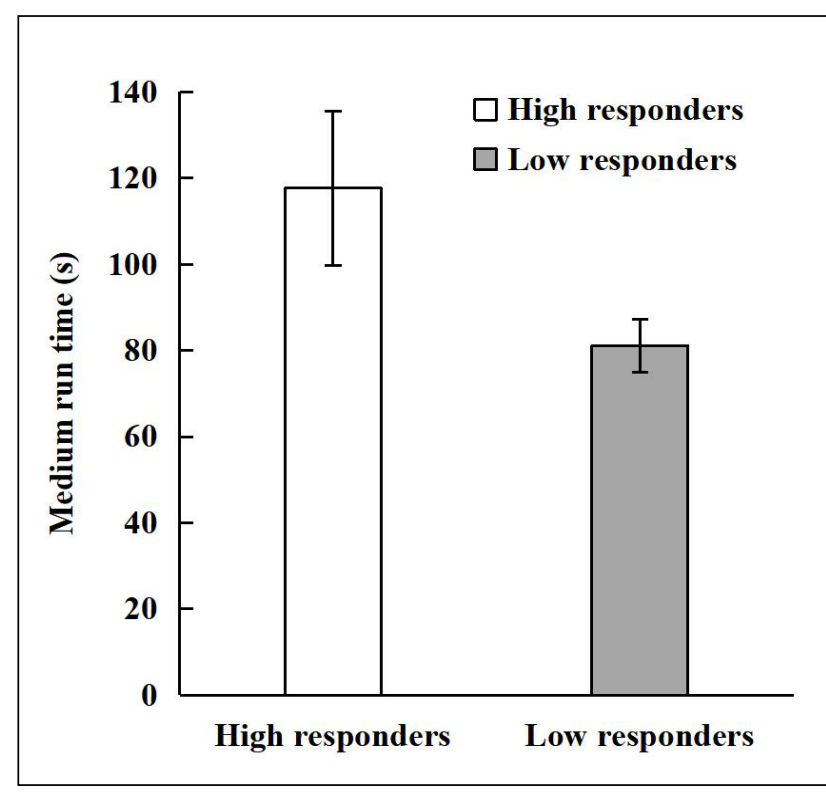

Figure 2. High Prevnar 13 antibody response mice showed an increased run time on the rotating rod test compared to the low antibody response group, $N=8-10$ per cohort, $P \leq 0.05$.

the high IgG antibody response group, as determined one month after vaccination, had a longer run time on the rotarod test (Figure 2) and a faster escape time in the spatial navigation learning task (Figure 3) compared to mice in the low antibody response group.

These observations in mice suggest that antibody levels to Prevnar 13, as measured by Elisa, could serve as a model for predicting age-related resilience. The translational relevance is potentially impactful because older adults are vaccinated with Prevnar 13, and retrospective and prospective studies could be developed to investigate the correlation of serum antibody levels with a variety of aging parameters.

\section{Declarations}

Cite this article as: Ryan $\mathrm{O}$, Zhou $\mathrm{J}$, Michali $\mathrm{I}$, et al. An immune stress test for resilience to aging: Pneumococcal vaccine response [J]. Aging Pathobiology and Therapeutics, 2020, 2(3): 171-172.

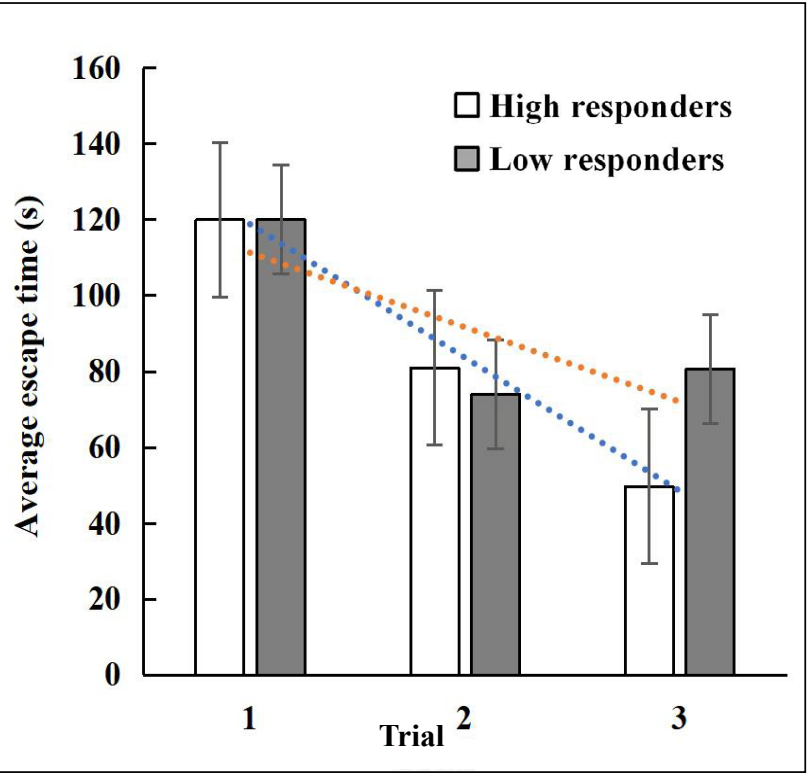

Figure 3. High Prevnar 13 antibody response mice showed a lower R2 slope for escape times in the box maze compared to the low antibody response group, $N=8-10$ per cohort. $P \leq 0.05$ for trial 3 .

Financial support and sponsorship: Supported by a grant from the National Institute on Aging, R01 AG057381 (W. Ladiges, PI).

Conflict of Interest: Warren Ladiges is a member of the Editorial Board of Aging Pathobiology and Therapeutics. All authors declare no conflict of interest and were not involved in the journal's review or desicions related to this

\section{References}

1. Kennedy B K, Berger S L, Brunet A, et al. Geroscience: linking aging to chronic disease. Cell, 2014, 159(4): 709713.

2. Kirkland J L, Stout M B, Sierra F. Resilience in aging mice. Journals of Gerontology Series A: Biomedical Sciences and Medical Sciences, 2016, 71(11): 1407-1414. manuscript. 